\title{
MONARCH-plus: the evidence of efficacy and safety of abemaciclib in countries with limited clinical research opportunities
}

\author{
Ahmad Awada, Andrea Gombos \\ Medical Oncology Department, Institut Jules Bordet, Université Libre de Bruxelles, Brussels, Belgium \\ Correspondence to: Ahmad Awada, MD, PhD. Medical Oncology Department, Institut Jules Bordet, Université Libre de Bruxelles, Brussels, Belgium. \\ Email: ahmad.awada@bordet.be. \\ Comment on: Zhang QY, Sun T, Yin YM, et al. MONARCH plus: abemaciclib plus endocrine therapy in women with HR+/HER2- advanced breast \\ cancer: the multinational randomized phase III study. Ther Adv Med Oncol 2020;12:1758835920963925.
}

Received: 27 August 2021; Accepted: 26 September 2021.

doi: $10.21037 /$ tbcr-21-25

View this article at: https://dx.doi.org/10.21037/tbcr-21-25

Estrogen receptor positive (ER+)/ human epidermal growth factor receptor 2 negative (HER2-) breast cancer is the most frequent subset, accounting for around $70 \%$ of all breast cancer cases (1).

Cyclin dependent kinase 4/6 (CDK 4/6) inhibitors (palbociclib, abemaciclib and ribociclib) combined with endocrine therapy (ET), non-steroidal aromatase inhibitors (NSAI) or fulvestrant became a standard treatment in ER+/ HER2- advanced breast cancer (ABC) and represents one of the major breakthrough in metastatic breast oncology during the past two decades. This is based on the substantial and clinically meaningful progression free survival (PFS) benefit seen in all trials conducted using CDK 4/6 inhibitors + ET [hazard ratio (HR): 0.55-0.56], the improvement in overall survival (OS) seen in several trials (HR: 0.72-0.75) and the good safety profile (2-10). Although some differences can be noticed in the inclusion criteria of these pivotal trials mainly regarding anticancer treatment history, results can be considered similar with the three different drugs and are consistent through all subgroups of patients. Main results and the most important characteristics of patient population included are summarized in Table 1. Current guidelines recommend that every advanced luminal breast cancer patient be treated with CDK 4/6 inhibitors (11).

Two of the above-mentioned pivotal phase III trials used abemaciclib (Verzenio ${ }^{\mathrm{TM}}$, Eli Lilly and Company, Indianapolis, IN, USA) + ET based combinations. MONARCH-2 enrolled 669 women with ER+/HER2$\mathrm{ABC}$ who had disease progression during prior ET. Patients were treated with fulvestrant plus either placebo $(n=223)$ or abemaciclib $150 \mathrm{mg}$ twice daily $(\mathrm{n}=446)$. Median PFS was prolonged from 9.3 to 16.4 months with abemaciclib plus fulvestrant (HR: $0.55 ; \mathrm{P}<0.001$ ), the response rate was $35.2 \%$ vs. $16.1 \%$ in the placebo group (7). The trial showed also a significant improvement in OS with the combination: 46.7 vs. 37.3 months (HR: $0.757, \mathrm{P}=0.01)(8)$.

MONARCH-3 was a phase III trial using abemaciclib $(n=328)$ or placebo $(n=165)$ plus an aromatase inhibitor (AI) in 493 postmenopausal women with ER+/HER2- ABC with endocrine-sensitive disease who haven't received any prior systemic therapy for ABC. Abemaciclib plus AI showed a significantly longer median PFS than the AI plus placebo (28.18 vs. 14.76 months; HR: $0.540, \mathrm{P}=0.000002)$. The response rate was $61 \%$ with abemaciclib and $45.5 \%$ with placebo $(\mathrm{P}=0.003)$ (12). Survival data are still immature.

Abemaciclib is the latest oral CDK 4/6 inhibitor to receive FDA approval, being granted breakthrough therapy designation in September 2017 as a 2nd-line treatment, combined with fulvestrant [+ luteinizing hormonereleasing hormone agonist (LHRHa) in pre-menopausal patients], for ER+/HER2 - metastatic breast cancer (mBC) who progressed on prior ET. In February 2018, abemaciclib received FDA approval as a 1st-line treatment in combination with an AI. Contrary to other CDK 4/6 inhibitors, is also approved as a single agent $(200 \mathrm{mg}$ twice daily) for ER+/HER2 - mBC who progressed after prior chemotherapy and ET (13).

In the current issue of Translational Breast Cancer 


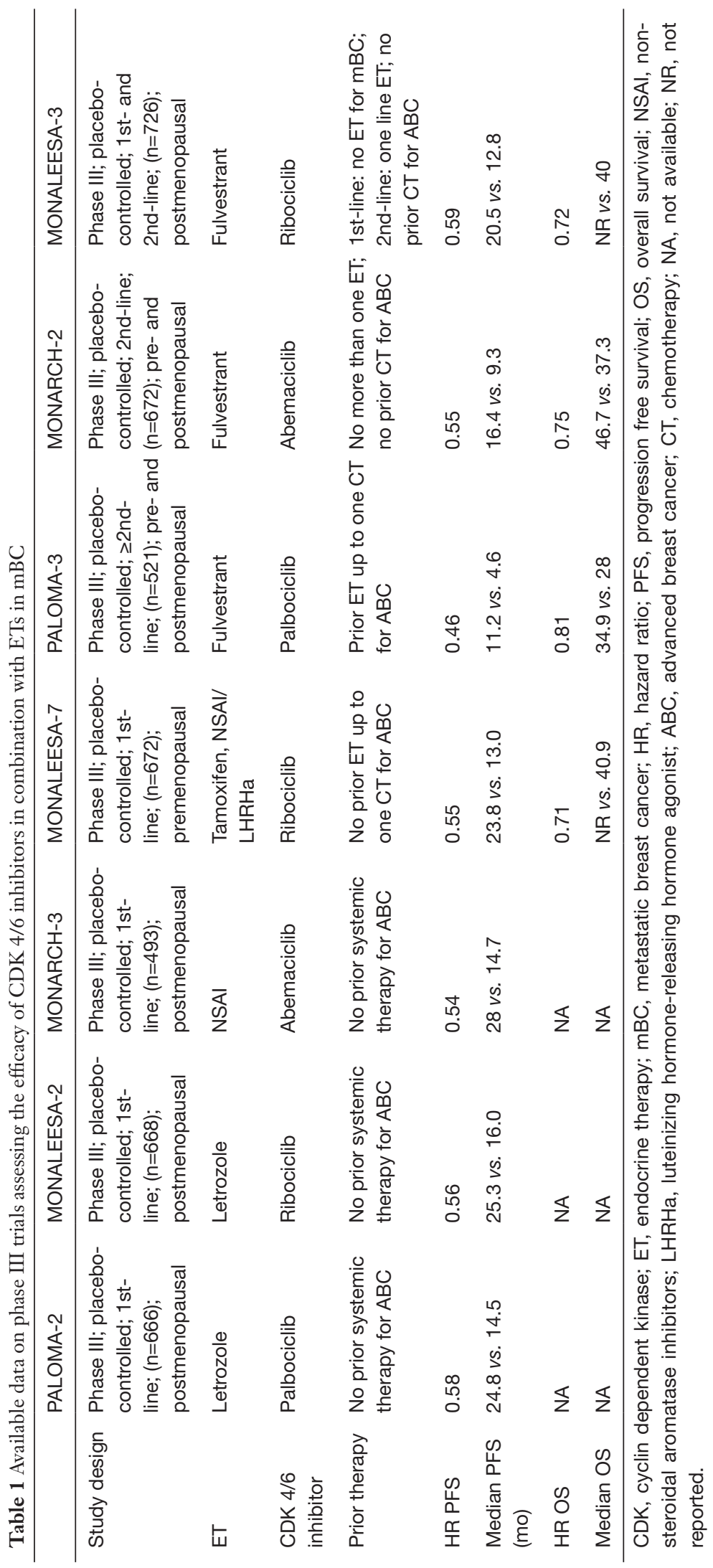


Research, Zhang et al. reports a pre-specified interim analysis of a randomized, double blind phase III trial conducted in China, Brazil, India and South Africa assessing the efficacy and safety of the combination of abemaciclib with either AI (cohort A, n=306) or fulvestrant (cohort B, n=157) compared to ET alone (14). Patients in cohort A had no prior systemic therapy for advanced/recurrent breast cancer and relapsed $>1$ year after adjuvant $\mathrm{AI}$ (if received), whereas patients in cohort B were refractory to NSAI (adjuvant or 1st-line metastatic setting) and had no prior chemotherapy for metastatic disease. Sixty percent of the patients had visceral involvement. Only $10 \%$ had prior $\mathrm{AI}$ and $40 \%$ did not received adjuvant ET in cohort A. As per inclusion criteria, almost all patients $(98.7 \%)$ in cohort B were previously treated with an $\mathrm{AI}$ and $35 \%$ of them showed primary resistance according to $\mathrm{ABC}$ consensus guideline definition (11). Enrollment took place between December 2016 and August 2018 and most of the participating patients are originating from China $(80 \%$ in cohort $\mathrm{A}$ and $85 \%$ in cohort B).

This paper reports an interim analysis after 119 events of the primary endpoint with a median follow-up up of 16 months (investigator assessed PFS in cohort A). Moreover, data on a number of secondary endpoints such as PFS in cohort $\mathrm{B}$, objective response rate (ORR), disease control rate (DCR), clinical benefit rate (CBR) and safety are included.

Median PFS was not reached in the abemaciclib arm and it was 14.7 months in the placebo arm [HR: 0.499, $95 \%$ confidence interval (CI): $0.346-0.719, \mathrm{P}=0.0001]$. In cohort $\mathrm{B}, 82$ events occurred at the time of this interim analysis, median PFS was also significantly better in the abemaciclib arm: 11.5 vs. 5.6 months (HR: 0.376, 95\% CI: $0.240-0.588, \mathrm{P}<0.0001)$. As in other pivotal trials, a significant improvement was observed with abemaciclib in all secondary endpoints such as ORR, DCR and CBR. The authors report a subgroup analysis, which show no differential efficacy of abemaciclib in relation to disease characteristics. Nevertheless, this analysis should be interpreted with caution given the small number of patients in each group.

In terms of safety profile, patients receiving abemaciclib experienced higher rates of neutropenia $[80 \%, 30 \%$ grade 3 (G3)], diarrhea (80\%, 4\% G3), anemia (62\%, 11\% G3), thrombocytopenia (44\%, 5.4\% G3), liver function test and blood creatinine increase (35\% and $12 \%$ respectively). A surprisingly high rate of pneumonitis was observed in both arms $(6.3 \%$ with abemaciclib and $3 \%$ with ET alone). Venous thromboembolic events occurred in $2 \%$ and $3.8 \%$ of patients treated with abemaciclib in cohorts $\mathrm{A}$ and $\mathrm{B}$ respectively. Of note, treatment discontinuation due to adverse events was $10.7 \%$ in cohort $\mathrm{A}$ and $3.8 \%$ in cohort B.

The main strength of MONARCH-plus is that it has been conducted in countries with much limited clinical research opportunities and particularly in a population which was underrepresented in pivotal phase III trials. Data reassures oncology community that abemaciclib has potentially the same activity in these populations. Furthermore, and of importance no signals of differential toxicity profile were seen. The real-life data can be used to report antitumor activity of new drugs in specific patient populations such as patients in specific countries. This approach suffers from a substantial clinical and statistical bias. MONARCH-plus is a more valid approach to document activity and mainly safety profile. Hopefully, these results can foster the availability of CDK $4 / 6$ inhibitors in these and similar countries for all ER+/HER2 breast cancer patients.

\section{Acknowledgments}

Funding: None.

\section{Footnote}

Provenance and Peer Review: This article was commissioned by the editorial office, Translational Breast Cancer Research. The article did not undergo external peer review.

Conflicts of Interest: Both authors have completed the ICMJE uniform disclosure form (available at https://tbcr. amegroups.com/article/view/10.21037/tbcr-21-25/coif). AA reports that he receives grants and takes participation on a Data Safety Monitoring Board or Advisory Board held by Roche, Lilly, Amgen, EISAI, BMS, Pfizer, Novartis, MSD, Genomic Health, Ipsen, AstraZeneca, Bayer, Leo Pharma, Merck, and Daiichi. AG reports advisory fees from Lilly, and Daiichi Sankyo, honoraria for presentation from Daiichi Sankyo, support for attending meetings from Pfizer. The authors have no other conflicts of interest to declare.

Ethical Statement: The authors are accountable for all aspects of the work in ensuring that questions related to the accuracy or integrity of any part of the work are appropriately investigated and resolved. 
Open Access Statement: This is an Open Access article distributed in accordance with the Creative Commons Attribution-NonCommercial-NoDerivs 4.0 International License (CC BY-NC-ND 4.0), which permits the noncommercial replication and distribution of the article with the strict proviso that no changes or edits are made and the original work is properly cited (including links to both the formal publication through the relevant DOI and the license). See: https://creativecommons.org/licenses/by-nc-nd/4.0/.

\section{References}

1. Howlader N, Altekruse SF, Li CI, et al. US incidence of breast cancer subtypes defined by joint hormone receptor and HER2 status. J Natl Cancer Inst 2014;106:dju055.

2. Finn RS, Martin M, Rugo HS, et al. Palbociclib and letrozole in advanced breast cancer. $\mathrm{N}$ Engl J Med 2016;375:1925-36.

3. Turner NC, Ro J, André F, et al. Palbociclib in hormonereceptor-positive advanced breast cancer. $\mathrm{N}$ Engl J Med 2015;373:209-19.

4. Hortobagyi GN, Stemmer SM, Burris HA, et al. Ribociclib as first-line therapy for HR-positive, advanced breast cancer. N Engl J Med 2016;375:1738-48.

5. Slamon DJ, Neven P, Chia S, et al. Phase III randomized study of ribociclib and fulvestrant in hormone receptorpositive, human epidermal growth factor receptor 2-negative advanced breast cancer: MONALEESA-3. J Clin Oncol 2018;36:2465-72.

6. Goetz MP, Toi M, Campone M, et al. MONARCH 3: Abemaciclib as initial therapy for advanced breast cancer. J Clin Oncol 2017;35:3638-46.

7. Sledge GW Jr, Toi M, Neven P, et al. MONARCH 2:

doi: $10.21037 /$ tbcr-21-25

Cite this article as: Awada A, Gombos A. MONARCH-plus: the evidence of efficacy and safety of abemaciclib in countries with limited clinical research opportunities. Transl Breast Cancer Res 2022;3:5.
Abemaciclib in combination with fulvestrant in women with HR+/HER2- advanced breast cancer who had progressed while receiving endocrine therapy. J Clin Oncol 2017;35:2875-84.

8. Sledge GW Jr, Toi M, Neven P, et al. The effect of abemaciclib plus fulvestrant on overall survival in hormone receptor-positive, ERBB2-negative breast cancer that progressed on endocrine therapy-MONARCH 2: a randomized clinical trial. JAMA Oncol 2020;6:116-24.

9. Turner NC, Slamon DJ, Ro J, et al. Overall survival with palbociclib and fulvestrant in advanced breast cancer. $\mathrm{N}$ Engl J Med 2018;379:1926-36.

10. Slamon DJ, Neven P, Chia S, et al. Overall survival with ribociclib plus fulvestrant in advanced breast cancer. $\mathrm{N}$ Engl J Med 2020;382:514-24.

11. Cardoso F, Paluch-Shimon S, Senkus E, et al. 5th ESOESMO international consensus guidelines for advanced breast cancer (ABC 5). Ann Oncol 2020;31:1623-49.

12. Johnston S, Martin M, Di Leo A, et al. MONARCH 3 final PFS: a randomized study of abemaciclib as initial therapy for advanced breast cancer. NPJ Breast Cancer 2019;5:5.

13. FDA. FDA approves new treatment for certain advanced or metastatic breast cancers. 2017 [cited $2021 \mathrm{Jul}$ 1]. Available online: https://www.fda.gov/news-events/pressannouncements/fda-approves-new-treatment-certainadvanced-or-metastatic-breast-cancers

14. Zhang QY, Sun T, Yin YM, et al. MONARCH plus: abemaciclib plus endocrine therapy in women with HR+/HER2- advanced breast cancer: the multinational randomized phase III study. Ther Adv Med Oncol 2020;12:1758835920963925. 